\title{
Real-Time Geometric Distortion Correction for Interventional Imaging with Echo-Planar Imaging (EPI)
}

\author{
Iulius Dragonu, ${ }^{1}$ Baudouin Denis de Senneville, ${ }^{1,2}$ Bruno Quesson, ${ }^{1}$ Chrit Moonen, ${ }^{1}$ and \\ Mario Ries ${ }^{1 *}$
}

\begin{abstract}
Many MR-guided interventional procedures rely on fast imaging sequences for providing images in real-time with a precise relation between the target position in the image and its true position. Echo-planar imaging (EPI) methods are very fast but prone to geometric distortions. Here, we propose a correction method designed for real-time conditions, adapting existing approaches based on dual EPI acquisition with varying echo times. The method is demonstrated with MR-thermometry for guiding thermal therapies. The proposed approach imposes a small penalty in acquisition speed but adds negligible latency to data processing, an important element for interventions of mobile organs. Magn Reson Med 00:000-000, 2009. (c) 2008 Wiley-Liss, Inc.
\end{abstract}

In typical interventional procedures such as catheter tracking, MR-guided biopsies, or MR-guided thermal ablation, rapid MR-imaging is typically used to provide functional or positional information in real-time, which is used to retroactively control the interventional procedure. In general, this requires rapid MR-sequences coupled with fast data processing strategies to achieve high frame-rates and low image latencies, allowing guidance of the interventional process and resolution of physiological motion.

Although a large variety of sequences, such as multiecho balanced steady-state free precession (TrueFISP) (1) or single-shot echo-planar-imaging (EPI) (2), have been used for this purpose, many rely on EPI readout trains for rapid data acquisition $(3,4)$. However, EPI images suffer from geometric distortions due to off-resonance effects resulting from static magnetic field $\left(B_{0}\right)$ inhomogeneities, from susceptibility changes between different anatomical regions especially near tissue/air and tissue/bone interfaces and from eddy-current effects. These distortions may lead to positional errors if the images are used for guiding external interventional devices.

Several correction techniques for geometric distortions have been proposed in the past, such as field inhomoge-

${ }_{1}^{1}$ Laboratory for Molecular and Functional Imaging: From Physiology to Therapy, Unité Mixte de Recherche (UMR) 5231 Centre National de la Recherche Scientifique (CNRS)/Université Bordeaux 2, Bordeaux, France.

2Institut Universitaire de Technologies (IUT) Bordeaux, Département Informatique, Gradignan, France.

Grant sponsor: Diagnostic Molecular Imaging; Grant number: EC-FP6-project LSHB-CT-2005-512146; Grant sponsors: Ligue Nationale Contre le Cancer; Conseil Régional d'Aquitaine; Ministère de la Recherche; Philips Medical System.

Current address for lulius Dragonu: Department of Diagnostic Radiology, Medical Physics, University Hospital Freiburg, Hugstetter Strasse 55, D-79106 Freiburg, Germany.

*Correspondence to: Mario Ries, UMR 5231, Imagerie Moléculaire et Fonctionnelle, Université «Victor Segalen» Bordeaux 2, 146, rue Leo Saignat, case 117, 33076 Bordeaux, France. E-mail: mario.ries@imf.u-bordeaux2.fr

DOI 10.1002/mrm.21903

Published online in Wiley InterScience (www.interscience.wiley.com).

(c) 2008 Wiley-Liss, Inc. neity mapping $(5,6)$, point-spread function calculation (7), or dynamically switched phase ramp in kt-space (8). The main limitations of these methods arise either from the inability to rapidly update the distortion information during the acquisition or from the introduced additional computational overhead, which may render them unsuitable for real-time image processing. Another interesting $B_{0}$ mapping-based approach was recently proposed by Priest et al. (9) using two reduced acquisitions interleaved (TRAIL). This method collects correction data by acquiring dual-echo images with halved image resolution. However, this method requires for the reconstruction the combination of nonsequentially-acquired half-resolution images, which can be a limiting factor in resolving rapid motion. Furthermore, TRAIL suffers from an intrinsic signal loss of $\sqrt{2}$, which is particularly limiting for interventional applications in regions with short $T_{2}^{*}$ such as abdomen or thorax.

We propose a distortion-correction based on field maps, as suggested by Reber et al. (6) in combination with a dynamic update strategy for the correction data and timeefficient data processing, as a simple and efficient method for the real-time correction of geometric distortions in gradient-recalled EPI images. The field maps are temporally updated to compensate for dynamic changes induced by physiological motion or the displacement of interventional devices, by continuously acquiring EPI images with alternating echo-time (TE) values. The proposed approach does not require additional acquisitions, and all the acquired images are used to correct for geometric distortions on the fly.

The proposed method may be particularly useful for MR-guided thermal therapies that induce local hyperthermia using laser, microwave, radio frequency (RF), or highintensity focused ultrasound $(10,11)$. In these applications MRI provides positional and temperature information $(12,13)$, and relies on gradient-echo imaging with long TE, often leading to severe distortion artifacts. This study demonstrates the possibility to remove severe image distortions dynamically with MR-thermometry in an ex-vivo RF-heating experiment, and its ability to remove EPI distortions in real-time in an in-vivo experiment imaging on the abdomen of a healthy volunteer under free-breathing conditions.

\section{THEORY}

For the case of a typical EPI readout train, the distortion in the read direction is small compared to the distortion in the phase-encoding direction:

$$
y_{p e}=y_{0 p e}+\frac{\Delta f\left(y_{0 p e}\right)}{B W_{p e}}
$$


where $y_{p e}$ is the distorted position in the EPI image, $y_{0 p e}$ is the correct position, $\Delta f\left(y_{0 p e}\right)$ is the magnetic field inhomogeneity, and $B W_{p e}$ is the bandwidth per pixel in the phase encode direction.

A field map can be determined by collecting two gradient echo images with different $T E$ values. After calculation of the phase maps from each image followed by phase unwrapping, the field map can be calculated from the phase difference $\Delta \phi$ :

$$
\Delta B_{0}=\frac{\Delta \phi}{2 \pi \cdot \not \cdot \Delta T E}
$$

where $₹$ is the gyromagnetic ratio in Hz/Tesla, and $\Delta T E=$ $\left(T E_{2}-T E_{1}\right)$ with $T E_{1,2}$ representing the alternating echotimes, respectively.

Furthermore, the $B_{0}$ map can be converted into a pixel shift in the phase-encoding direction $(\Delta y)$ according to the following equation:

$$
\Delta y=\frac{\not \cdot \Delta B_{0}}{B W_{p e}}
$$

Making use of Eqs. [2] and [3], one can derive the value of the pixel shift directly from the unwrapped phase difference according to the following relation:

$$
\Delta y=\frac{\Delta \phi}{2 \pi \cdot B W_{p e} \cdot \Delta T E}
$$

Two subsequent images are combined in a sliding window to update the field map dynamically for each new acquired image. This leads under the Nyquist criteria to a temporal resolution of $2 T_{a c q}$ for the correction, where $T_{a c q}$ represents the acquisition time of the EPI image. Note that the modifications of the magnetic susceptibility field induced by organ motion during the acquisition of the two subsequent images have not been taken into account in Eq. [4]. Alternatively, a linear interpolation is proposed between the phases $\left(\phi_{T E 1(n-1)}\right.$ and $\left.\phi_{T E 1(n+1)}\right)$ of two consecutive images with the same TE. This linearly interpolated value gives a more accurate representation of the pixel shift at instant $t_{n}$ according to the following relation:

$$
\begin{aligned}
\left.\Delta y=\frac{1}{2 \pi \cdot B W_{p e} \cdot\left(T E_{2}\right.}-T E_{1}\right) & \\
& \times\left(\phi_{T E 2(n)}-\frac{\left.\phi_{T E 1(n-1)}+\phi_{T E 1(n+1)}\right)}{2}\right)
\end{aligned}
$$

However, this approach has the inconvenience to introduce a latency of one image to the correction and to reduce the temporal resolution from $2 T_{a c q}$ to $3 T_{a c q}$.

For the use of the correction in interventional procedures, it is important to evaluate the upper bound of the precision using Eq. [4]:

$$
\sigma(\Delta y)=\frac{1}{2 \pi \cdot B W_{p e} \cdot \Delta T E} \sqrt{\sigma^{2}\left(\phi_{T E 1}\right)+\sigma^{2}\left(\phi_{T E 2}\right)}
$$

Conturo et al. (14) have demonstrated that the phase error is inversely proportional to the signal-to-noise ratio (SNR) of the complex MR image, i.e. $\sigma(\phi)=\sigma(|I|) /|I|=$ 1/SNR, where $I$ and $\sigma(|I|)$ represent the intensity and the noise level of the magnitude image, respectively. Assuming the same noise level in both EPI images and taking into account the $T_{2}^{*}$ signal decrease, we derive the following equation for estimating the precision of the displacement value:

$\sigma(\Delta y)$

$$
=\frac{1}{2 \pi \cdot B W_{p e}} \cdot \frac{1}{S N R\left(T E_{\text {short }}\right)} \cdot \frac{1}{\Delta T E} \cdot \sqrt{1+\exp \left(\frac{2 \Delta T E}{T^{*}{ }_{2}}\right)}
$$

where, $\operatorname{SNR}\left(T E_{\text {short }}\right)$ represents the SNR of the EPI image acquired with the short echo-time $\left(T E_{\text {short }}\right)$.

\section{MATERIALS AND METHODS}

\section{Data Acquisition and Processing}

All experiments were performed on a 1.5T Philips Achieva clinical scanner using a modified gradient-recalled EPI sequence allowing the acquisition of dynamic images with alternating $T E$ values. In a first processing step, the pixelby-pixel phase difference of two subsequent images is calculated and a temporal phase unwrapping step is performed to remove $2 \pi$ aliasing. Then, the displacement of each pixel in the phase encoding direction is directly calculated using Eq. [5]. Finally, this information is used to unwrap the current EPI image. The distorted EPI images can be considered as irregularly sampled data, which is mapped on a regularly-sampled Cartesian grid. Adjacent pixels on the irregular grid were remapped and linearly interpolated to obtain the pixel intensities on the Cartesian grid. To avoid interpolation between regions with very low signal intensity and object boundaries, an adaptive thresholding was applied previous to this step. For the in-vivo experiments with low SNR, an additional filtering step was introduced: the thresholding mask was obtained from a filtered version of the magnitude image (Gaussian filter, 3-pixel kernel diameter) and the distortion map was calculated from phase data derived from smoothed complex data (Gaussian filter, 3-pixel kernel diameter), whereas all geometric corrections were applied to the unfiltered complex data.

The unwrapping algorithm was applied to the complex pixel values. This allows undistorted magnitude and phase images to be obtained, which can be used for MRthermometry using the proton resonance frequency (PRF) shift technique $(12,13)$.

\section{Heating Study}

A fresh ex-vivo pig kidney was installed on a motorized platform performing a periodical linear displacement of $10 \mathrm{~mm}$ in $6.3 \mathrm{~s}$. Heating was performed with two copper electrodes using a clinical RF device (Tyco Healthcare/ Radionics, Burlington, MA, USA) operating at $480 \mathrm{kHz}$ frequency with $100 \mathrm{~W}$ of maximum output power. RF power at $10 \mathrm{~W}$ was applied $15 \mathrm{~s}$ from the start of the image sequence with a total duration of $22 \mathrm{~s}$. MRI was performed 
using the following single-shot EPI sequence: $1024 \mathrm{dy}$ namic coronal images, $T R=57 \mathrm{~ms}, T E=27 \mathrm{~ms}, \Delta T E=$ $3 \mathrm{~ms}$, flip angle $=25^{\circ}, \mathrm{FOV}=240 \times 128 \mathrm{~mm}^{2}$, slice thickness $=6 \mathrm{~mm}$, matrix size $=112 \times 60, B W_{p e}=$ $22.4 \mathrm{~Hz}$, and $B W_{r d}=2593.4 \mathrm{~Hz}$. A $T_{2}$-weighted turbo spin-echo (TSE) sequence with the same resolution was used for comparison.

Motion-compensated MR-thermometry was performed as presented (15). This PRF-based method acquires complete set of reference magnitude and phase images for each organ position during a pretreatment step. During the intervention, the current magnitude image is compared to the prerecorded set and the closest match selected by evaluating the intercorrelation coefficient. The corresponding prerecorded phase image serves as reference for current temperature computation. This strategy allows correcting online periodical interscan motion-related errors in PRF temperature methods. To be compatible with the proposed distortion correction, the two separate lookup tables $(N=100)$ for each of the two $T E$ values were used.

\section{Real-Time In-Vivo Study}

Dynamic MRI was performed under free-breathing conditions of the abdomen of a healthy volunteer and reconstructed in real-time. The MR sequence employed the following parameters: 500 dynamic sagittal single-shot EPI images, $T R=78 \mathrm{~ms}, T E=39 \mathrm{~ms}, \Delta T E=7 \mathrm{~ms}$, FOV $=$ $350 \times 227.5 \mathrm{~mm}^{2}$, slice thickness $=7 \mathrm{~mm}$, matrix size $=$ $128 \times 84, B W_{p e}=14.1 \mathrm{~Hz}$, and $B W_{r d}=2435 \mathrm{~Hz}$. To avoid chemical shift artifacts in the field map due to fat resonance frequency, a 121 spectral-spatial (16) water selective pulse was used. A four-channel abdominal phased-array coil was used for signal detection. The experiment was repeated with a fixed echo-time in order to evaluate the influence of modifications of the magnetic susceptibility field induced by organ motion during the acquisition of two subsequent images. This correction error can be calculated by inserting the phase differences into Eq. [3].

$k$-Space data was transferred directly from the MR acquisition system to an external in-house-developed realtime reconstructor using The Ace Orb (TAO) a real-time implementation of the Common Object Request Broker Architecture (CORBA) communication protocol (17). The entire reconstruction was implemented as a multithreaded processing chain in $\mathrm{C}++/$ OpenMP on a four-processor AMD Opteron $(2.4 \mathrm{GHz})$. The bulk reconstruction time per image was $4 \mathrm{~ms}$ and the image latency was $55 \mathrm{~ms}$ post-TE.

A steady-state free precession (SSFP) image with the same image geometry and a two-fold increased image resolution was used for the evaluation of the efficacy of the correction $(T R=3.3 \mathrm{~ms}, T E=1.66 \mathrm{~ms})$.

\section{RESULTS}

\section{Theoretical Calculations}

Figure 1a shows the theoretical precision of the correction given by Eq. [7], evaluated for the typical case of an EPI sequence, as a function of $\triangle T E$, in pixels per unitary bandwidth. The function was evaluated for two SNR values (10 and 15), which typically represents the lower limit for MR-thermometry or interventional guidance and for the $T_{2}^{*}$ values for liver and kidney tissue at 1.5T (18). The minima of this function can be found for a $\Delta T E$ which is equal to 1.108 times the $T_{2}^{*}$ of the observed tissue. However, in practice a shorter $\triangle T E$ is preferable for the correction to avoid phase aliasing due to strong local field deviation and to limit the time penalty on the acquisition speed of the sequence. Assuming a precision of less than one-third of a
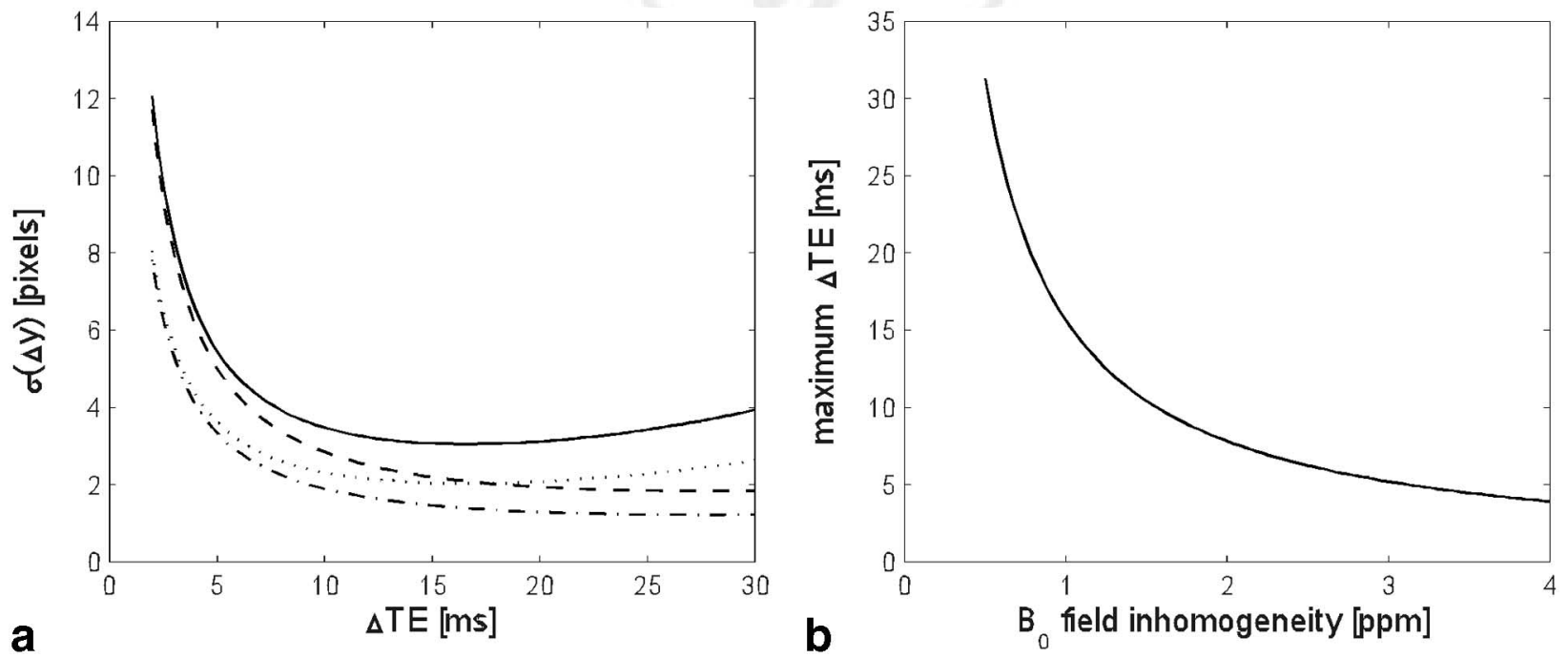

FIG. 1. a: Theoretical values of the uncertainty of the displacement vectors normalized to an acquisition bandwidth in the phase encoding direction of $1 \mathrm{~Hz}$ as a function of $\Delta \mathrm{TE}$. The error of the displacement can be computed by dividing the vertical axis number from the graph by the bandwidth in the phase-encoding direction. The error is shown for two different $T_{2}^{\star}$ values and two different SNRs. The solid line and dotted line represent the correction uncertainty for liver tissue ( $T_{2}^{*}=15 \mathrm{~ms}$ at $1.5 \mathrm{~T}$ ) for an SNR of 10 (solid) and 15 (dotted), respectively. The dashed line and the dashed-dotted line correspond to the values typically found in kidney tissue ( $T_{2}^{\star}=25$ ms at $\left.1.5 T\right)$ for an SNR of 10 (dashed) and 15 (dash-dotted), respectively. b: Upper limit for $\Delta T E$ before phase aliasing occurs as a function of the magnetic field inhomogeneity for a $B_{0}$ field of $1.5 \mathrm{~T}$ in a given region of interest. 
pixel as the acceptance criteria for the correction, and considering the most unfavorable case: a SNR value of 10 , the lowest EPI phase-encoding bandwidth of the presented experiments of $14 \mathrm{~Hz}$ and the $T_{2}^{*}$ value of $15 \mathrm{~ms}$ corresponding to the liver tissue, a lower bound for $\triangle T E$ of $6 \mathrm{~ms}$ can be estimated as sufficient.

Strong local inhomogeneities can lead to phase differences larger than $2 \pi$ between subsequent phase maps and thus an incorrect displacement vector is calculated. To avoid this effect, $\triangle T E$ has to be chosen according to the largest field deviation that is present in the area of interest with the help of Eq. [2], as shown in Fig. 1b. For $\Delta T E=$ $5 \mathrm{~ms}$ to $7 \mathrm{~ms}$ the maximum inhomogeneity that can be corrected is $3.1 \mathrm{ppm}$ to $2.2 \mathrm{ppm}$ at $1.5 \mathrm{~T}$. For a typical $B W_{p e}$ $=22 \mathrm{~Hz}$ and $\Delta T E=5 \mathrm{~ms}$, distortions up to 9.1 pixels (i.e., \pm 4.55 pixel from the undistorted position) can be corrected. Considering the voxel size for the phantom study $\left(1.72 \times 1.72 \times 5 \mathrm{~mm}^{3}\right)$ the proposed method will correct distortions in the phase-encoding direction up to $\pm 7.82 \mathrm{~mm}$ with a precision of $\pm 0.57 \mathrm{~mm}$.

\section{Heating Study}

The accuracy of the correction is shown in Fig. 2. An edge detection algorithm was used to highlight the borders of the pig kidney in the TSE image (Fig. 2a). This information a

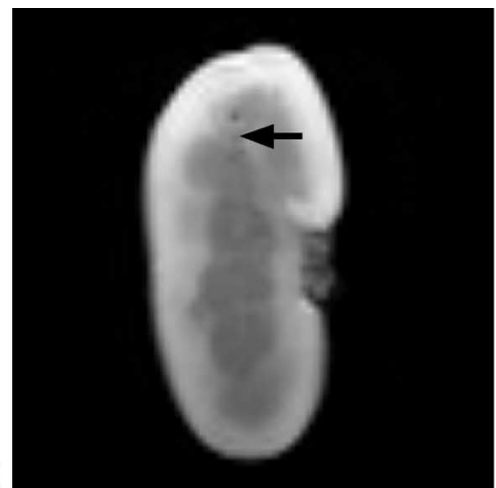

b

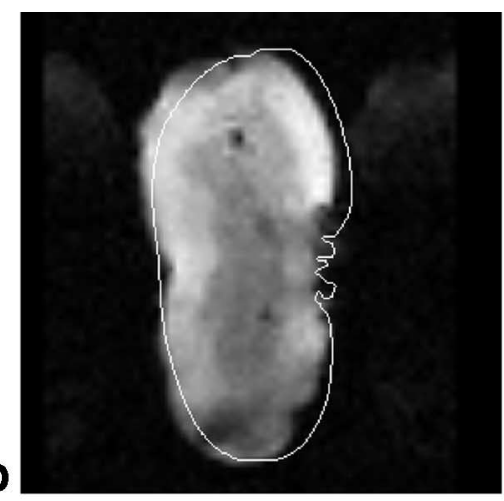

c
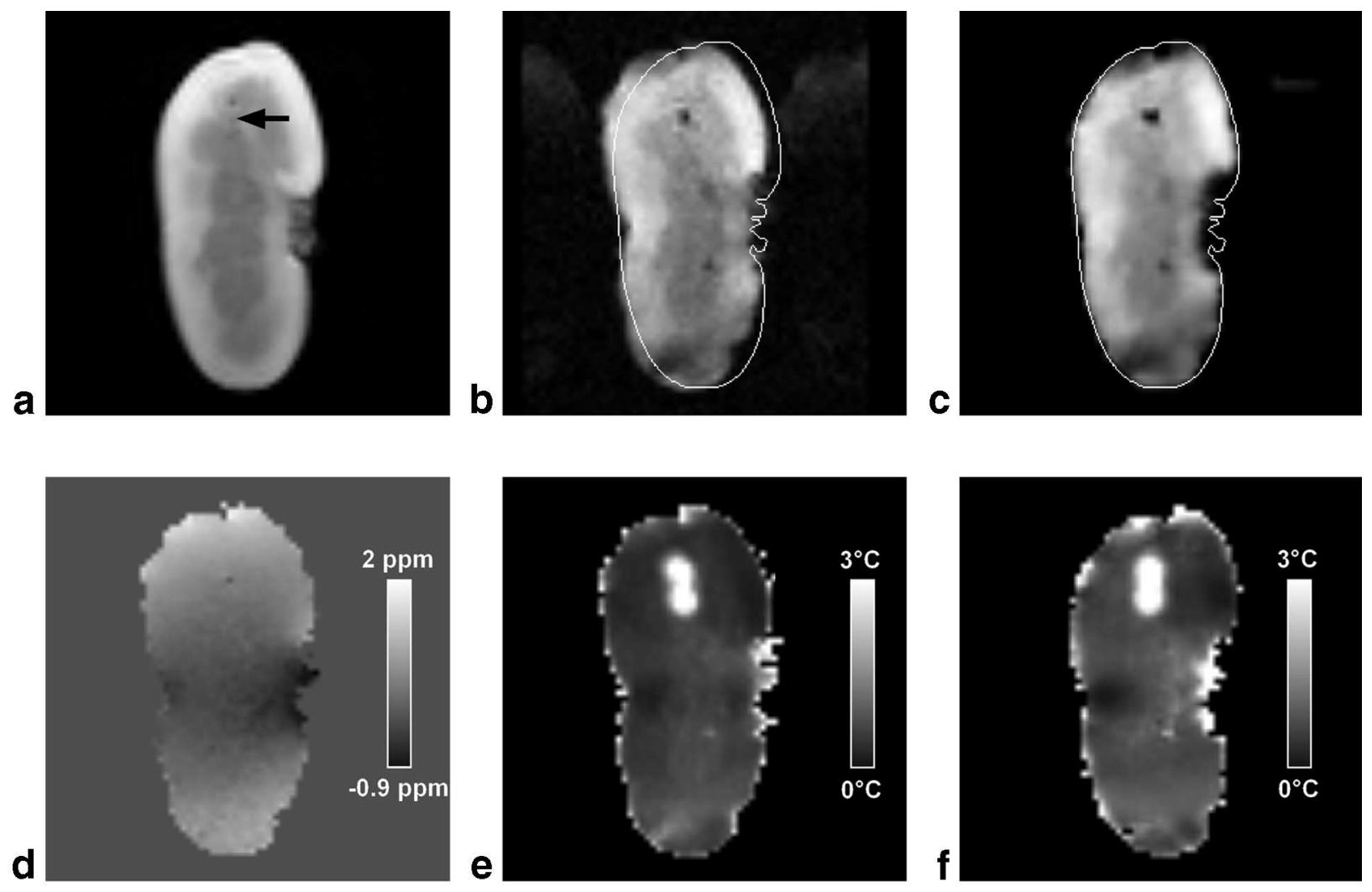
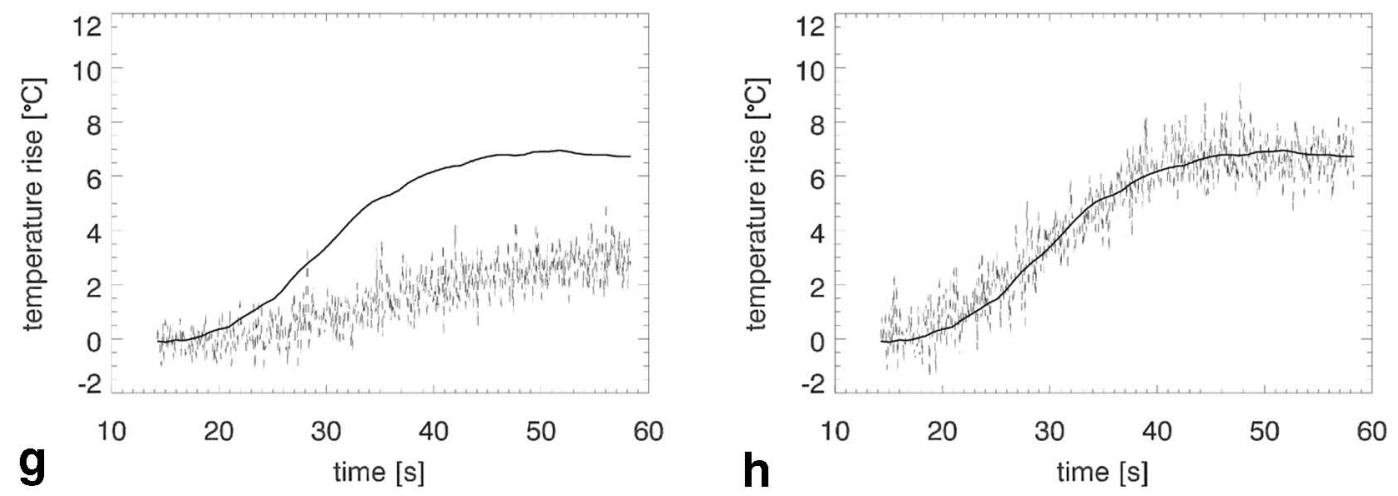

FIG. 2. Demonstration of the effectiveness of the proposed method for the ex-vivo heating study. a: TSE image used as a reference. b: Uncorrected EPI image. c: Corrected EPI image. d: The field inhomogeneity conditions of the experiment. Standard deviation of the distorted (e) and corrected (f) temperature maps during the heating experiment. Comparison between the PRF-based MR thermometry (dashed line) and the reference temperature measured with the optical fiber thermometer (solid line) at the position determined on the TSE image (black arrow in (a)) without (g) and with (h) the proposed distortion correction method. 
was overlaid on the distorted image (Fig. 2b) and the corrected EPI datasets. $B_{0}$ inhomogeneities $>2.0 \mathrm{ppm}$ were found (Fig. 2d), leading to shifts of up to 5.7 pixels. Figure $2 \mathrm{e}$ and $\mathrm{f}$ show the standard deviation (SD) of the temperature maps before and after the unwrapping algorithm. The high-intensity spots on both images correspond to the heated region in the vicinity of the copper electrodes. Low values of $\mathrm{SD}\left(<0.9^{\circ} \mathrm{C}\right)$ of temperature maps in the nonheated regions show that the precision of the MR-thermometry is not altered by the spatial remapping.

The advantage of a geometry correction for multimodal imaging is illustrated by Fig. $2 \mathrm{~g}$ and $\mathrm{h}$, which show the single-voxel temperature evolutions obtained by MR thermometry compared to the readings of a fiber optic probe. Since the position of the fiber optic probe was determined by an undistorted high-resolution spin-echo (SE) image (at the position indicated by the arrow in Fig. 2a), the MR thermometry based on the uncorrected EPI shows a temperature difference due to a geometry mismatch. The readings obtained from the distortion-corrected EPI images taken at the probe position lead to a temperature evolution that closely matches that of the readings of the fiber optic probe. Note that the readings remain a good match throughout the entire motion cycle despite the fact that the distortions at the position of the probe vary temporally by $0.5 \mathrm{ppm}$.

\section{Real-Time In-Vivo Study}

Figure $3 \mathrm{~b}$ shows the amplitude of the distortions of the uncorrected sagittal EPI image displayed in Fig. 3c extracted from the dynamic acquisition. The map shows large local fluctuations especially in the vicinity of tissue boundaries. While the distortions present within the kidney and the back muscle are below the resolution of the EPI images for this experiment, distortions in the phaseencoding direction (in this case anterior-posterior) within the liver exceed $1 \mathrm{~cm}$. Since the lung volume and the location of the organs has a direct impact on the local magnetic field homogeneity, the distortion field changes dynamically throughout the respiratory cycle. In the vicinity of the area indicated by arrow II in Fig. 3c the amplitude of the distortion oscillates by up to $8 \mathrm{~mm}$ during respiration. The overlaid anatomical reference points of the uncorrected image (Fig. 3c) and those of the corrected image (Fig. 3d) demonstrate the efficacy of the correction.

The error induced by field modifications during the acquisition of two subsequent images for a pixel within the liver is shown for direct phase subtraction and phase averaging in Fig. 3e and f, respectively. Direct phase subtraction results in a peak-to-peak error of 1.9 pixels and an SD of 0.35 pixels. Note that the error is two-fold periodic: It is modulated with the respiratory frequency and oscillates in average by 0.4 pixels between two subsequent images. Phase averaging removes this effect and reduces the error to 1.1 pixels peak-to-peak and an SD of 0.2 pixels.

\section{DISCUSSION}

For typical gradient-recalled EPI images of abdominal organs such as liver and kidney, with typical SNR $>15$, the simulations show that the proposed distortion correction
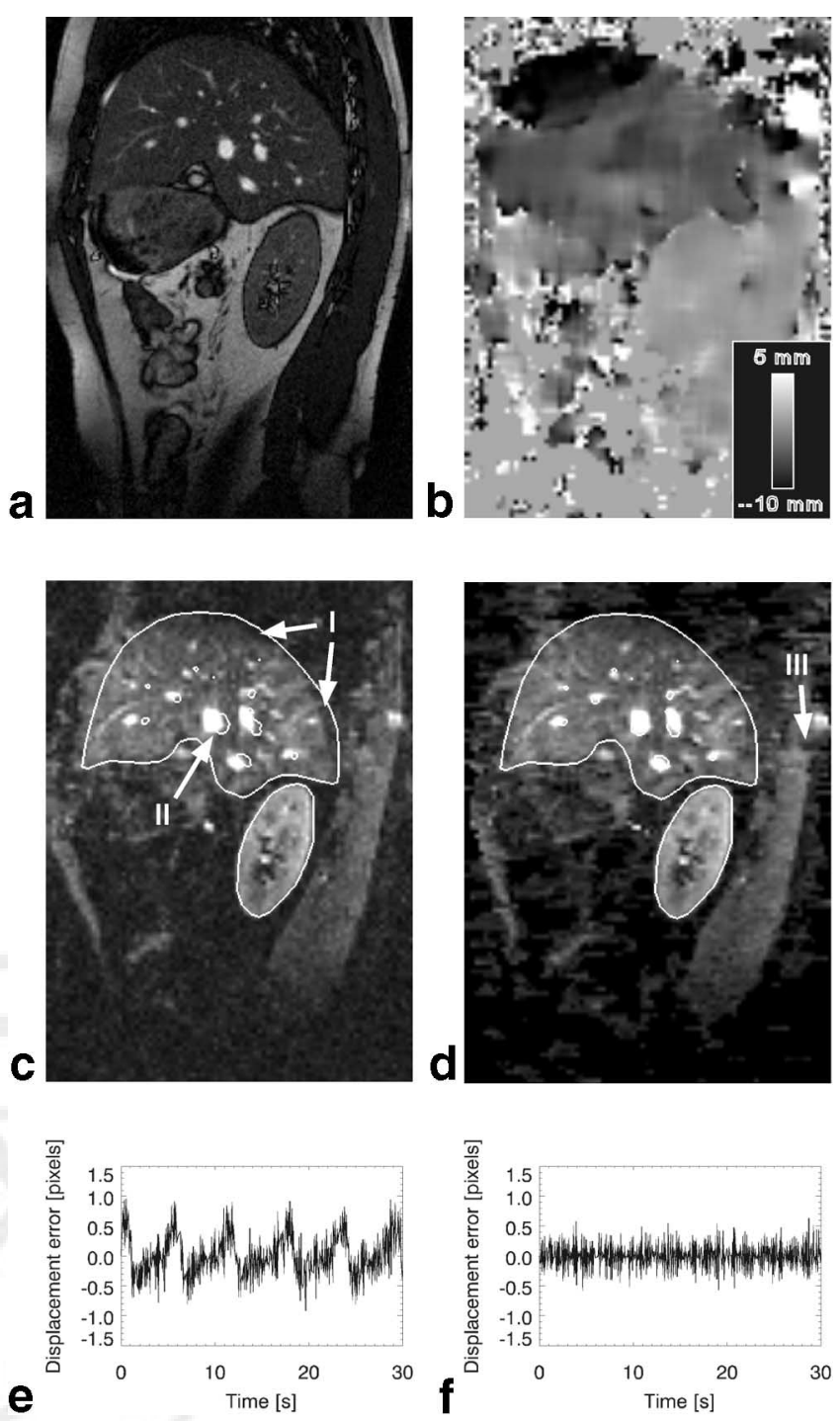

FIG. 3. a: A high-resolution SSFP image with a short TE was used as a reference image. $\mathbf{b}$ : A map of the distortions in millimeters $(\mathrm{mm})$ of the uncorrected EPI image displayed in (c), which is an example extracted from the temporal series. This distortion map is dynamically updated for each acquired image. The kidney shows moderate distortions of $\sim 1.5 \mathrm{~mm}$ in this example (below the voxel size), whereas image distortions in the liver can be larger than $1 \mathrm{~cm}$, especially in the vicinity of the diaphragm. The main anatomy of the liver and the kidney obtained from the SSFP image was transposed to the distorted EPI image (c) and the distortion-corrected EPI image (d) for comparison. Note the mismatch between the mask and the position of the vessels in the center of the liver and at the interface lung/liver (see arrow), which are removed by the distortion correction. The correction error at position indicated by arrow II for the direct phase subtraction (e) and for the phase-averaged technique (f).

results in a precision of the correction within 0.16 pixels while introducing an echo-time penalty of 5 to $7 \mathrm{~ms}$. The small echo-time penalty and the high precision correspond well with the real-time requirements of MR-guided interventional procedures.

The choice of $\triangle T E$ of $5 \mathrm{~ms}$ in the proposed implementation restricts the correction to areas with field variations 
less than $\pm 1.56 \mathrm{ppm}$ as shown in Fig. 1b. Areas with more important inhomogeneities therefore show an aliasing artifact of the correction, and thus need to be masked. For a typical single-shot EPI sequence with a $B W_{p e}$ of $14-22 \mathrm{~Hz}$ and a resolution of $2 \mathrm{~mm}$. this corresponds to shifts of $\pm 1.4 \mathrm{~cm}$ to $\pm 0.9 \mathrm{~cm}$. Since the proposed method is designed for real-time applications requiring low image latency and thus low computational overhead, this parameter choice represents a design compromise. This limitation can be circumvented by employing a 2D phase-unwrapping step to the phase images prior to the calculation of the field maps (19) as is required for TRAIL (9). However, this remains a computationally-intense processing step, which is often unstable in areas with signal discontinuities and with the strong susceptibility changes frequently encountered in abdominal imaging. In addition, these areas with strong susceptibility changes are subject to rapid intravoxel dephasing, which in turn leads to a low local SNR ( $\ll 10)$ for gradient-recalled imaging and are thus of limited value for MR-thermometry or MR-guidance (18).

Another limitation of the proposed correction method arises from the fact that the field maps are calculated using a sliding-window technique. When direct phase subtraction of subsequent images is employed during evolution of the field map, slight position and thus phase changes lead to small errors in the correction map. It can be shown with Eq. [4] that the sign of this residual error changes with every new correction map update. Consequently, the relative positioning error between two subsequently corrected images is effectively doubled. The in-vivo data reveals that this problem is in practice frequently encountered on fast-moving objects such as liver and kidney, as shown in Fig. 3e. The resulting dynamic correction artifact can be effectively removed by widening of the sliding window to three images in conjunction with phase averaging as shown in Fig. 3f. However, this reduces the temporal resolution of the correction to one-third of the bandwidth of the employed sequence and introduces a temporal latency of $1 T R$. Consequently, this type of correction is only suitable in conjunction with very fast sequences that observe the physiological motion of the target area with a very high temporal resolution.

The heating experiments demonstrate the compatibility of the proposed distortion correction with MR-thermometry during thermoablation. Furthermore, the distortions in the EPI images, introduced by the susceptibility difference between the RF electrodes and the surrounding tissue, are removed. In addition, the slight local frequency changes introduced by temperature variations in the observed tissue theoretically also lead to local image distortions of the EPI phase-encoded images. In the experiment presented, the total temperature difference in the vicinity of the RF needles was $28^{\circ} \mathrm{C}$. The resulting frequency shift can be calculated to $-0.28 \mathrm{ppm}$ (12). Using Eq. [4] with a $B W_{p e}=$ $22.4 \mathrm{~Hz}$, this leads to a shift of -0.81 pixel, which is superimposed on the distortions caused by the intrinsic magnetic field inhomogeneities. Since the proposed distortion correction dynamically updates the correction maps with each new arriving image, the spatial mismatch due to the temperature evolution can also be removed.

The in-vivo experiments presented demonstrate the ability to correct spatial distortions of EPI sequences for both organs in real-time with a dynamic correction scheme and allows a precise spatial match of thermometry with undistorted anatomical images. The absence of spatial fitting of the field map to polynomial or other model functions is advantageous for a real-time corrections, since this renders the method insensitive to signal discontinuities in regions where field maps cannot be determined, represents the local fluctuations well along organ boundaries compared to a globally-fitted function (5), and finally limits the required processing steps and thus processing time. The resulting low computational overhead leads to complete execution time of $1.5 \mathrm{~ms}$ for the entire correction (including the field calculation and the regridding of the complex data) and thus to reconstruction times below $5 \mathrm{~ms}$, making the method compatible with rapid acquisition sequences of more than 20 images per second. However, since the method collects correction data with one-half the temporal resolution of the base sequence, high frame rates are required to sample the changes during the motion cycle with sufficient resolution.

It should be noted that, if areas with large $B_{0}$ inhomogeneities lead to larger phase difference than $2 \pi$ during the chosen $\triangle T E$, the correction method will not only fail, but can also lead to increased distortions (see arrow III in Fig. $3 \mathrm{~d})$. It is thus indispensable that an initial experiment with a short $\triangle T E$ establishes a validity map for the subsequent correction with longer $\Delta T E$. In case the region of interest is not included in this validity map, either $\Delta T E$ has to be shortened accordingly with concomitant lower precision of the correction or $2 \mathrm{D}$ phase unwrapping has to be employed.

\section{CONCLUSIONS}

The proposed method is an extension of existing approaches based on dual-TE gradient-recalled images with long readout trains, and especially adapted for the use under real-time conditions for interventional imaging on moving targets. It is compatible with MR thermometry and interventional devices such as needles and catheters, which typically cause severe local image distortions.

The method proposed imposes a small speed penalty on the acquisition sequence but adds a negligible latency to the data processing, which is of great importance for realtime imaging. As a drawback, the method introduces a fluctuating signal magnitude and is therefore not suitable for applications that require the magnetization to remain in a steady-state, such as the observation of bolus passages or functional MRI (fMRI) experiments.

\section{REFERENCES}

1. Deimling M, Heid O. Magnetization prepared true FISP imaging. Proceedings of the 2nd Annual Meeting of ISMRM, San Francisco, CA, USA, 1994 (Abstract 495).

2. Mansfield P. Multi-planar image formation using NMR spin echoes. J Phys C 1977;10:L5-L58.

3. Pruessmann KP, Weiger M, Scheidegger MB, Boesiger P. SENSE: sensitivity encoding for fast MRI. Magn Reson Med 1999;42:952-962.

4. Sodickson DK, Manning WJ. Simultaneous acquisition of spatial harmonics (SMASH): fast imaging with radiofrequency coil arrays. Magn Reson Med 1997;38:591-603. 
5. Jezzard P, Balaban RS. Correction for geometric distortion in echo planar images from $B_{0}$ field variations. Magn Reson Med 1995;34:6573.

6. Reber PJ, Wong EC, Buxton RB, Franck LR. Correction of off resonancerelated distortion in echo-planar imaging using EPI-based field maps. Magn Reson Med 1998;39:328-330.

7. Zeng H, Constable RT. Image distortion correction in EPI: comparison of field mapping with point spread function mapping. Magn Reson Med 2002;48:137-146.

8. Xiang QS, Ye FQ. Correction for geometric distortion and N/2 ghosting in EPI by phase labeling for additional coordinate encoding (PLACE). Magn Reson Med 2007;57:731-741.

9. Priest AN, Phil D, De Vita E, Thomas DL, Ordidge RJ. EPI distortion correction from a simultaneously acquired distortion map using TRAIL. J Magn Reson Imaging 2006;23:597-603.

10. Kennedy JE, ter Haar GR, Cranston D. High intensity focused ultrasound: surgery of the future? Br J Radiol 2003;76:590-599.

11. Levy S. Biophysical basis and cardiac lesions caused by different techniques of cardiac arrhythmia ablation. Arch Mal Coeur Vaiss 1995; 88:1465-1469.
12. Hindman JC. Proton resonance shift of water in the gas and liquid states. J Chem Phys 1966;44:4582-4592.

13. Peters RD, Hinks RS, Henkelman RM. Ex-vivo tissue type independence in proton-resonance frequency shift MR thermometry. Magn Reson Med 1998;40:454-459.

14. Conturo TE, Smith GD. Signal-to-noise in phase angle reconstruction: dynamic range extension using phase reference offsets. Magn Reson Med 1990;15:420-437.

15. Denis de Senneville B, Mougenot C, Moonen CTW. Real time adaptive methods for treatment of mobile organs by MRI controlled high intensity focused ultrasound. Magn Reson Med 2007;57:319-330.

16. Meyer CH, Pauly JM, Macovski A, Nishimura DG. Simultaneous spatial and spectral selective excitation. Magn Reson Med 1983;54:539-542.

17. Pyrali I, Schmidt DC, Cytron R. Techniques for enhancing real-time CORBA quality of service. In: Proceedings of IEEE Real-Time Systems Symposium (RTSS), Cancun, Mexico, 2003;91:1070-1085.

18. Denis de Senneville B, Mougenot C, Quesson B, Dragonu I, Grenier N, Moonen CTW. MR thermometry for monitoring tumor ablation. Eur Radiol 2007;17: 2401-2410.

19. Dias JMB, Leitao JMN. InSAR phase unwrapping: a Bayesian approach. In: IEEE Geoscience and Remote Sensing Symposium. 2001;1:396-400. 